\title{
Graves' ophthalmopathy: I. Simple CT estimates of extraocular muscle volume
}

\author{
E SVERKER HALLIN' AND STEVEN E FELDON
}

From the 'Department of Ophthalmology, University of Göteborg, Sweden; and the ${ }^{2}$ Department of Ophthalmology and Neurological Surgery, University of Southern California School of Medicine, Los Angeles, California, USA

SUMMARY Graves' ophthalmopathy is an autoimmune inflammatory process affecting primarily the extraocular muscles. The enlargement of these muscles is responsible for most of the important manifestations of the disease, including proptosis, lid retraction, diplopia, and optic neuropathy. Recently the optic neuropathy has been shown to correlate with extraocular muscle volume as determined by computed tomography (CT). In the present study simplified measures of the extraocular muscles imaged by $\mathrm{CT}$ are described, which also correlate highly with the development of optic neuropathy.

Recently Feldon and coworkers' described a method for calculating the extraocular muscle volume of patients with Graves' ophthalmopathy, using high resolution computed tomographic sections. They demonstrated that the risk of developing optic nerve compression increased with extraocular muscle size and diminution of function. ${ }^{23}$ Unfortunately the time and equipment necessary to calculate extraocular muscle volume limited the general application of this technique. The purpose of this paper is to explore alternative methods of determining extraocular muscle volume that might be more easily accomplished in the clinical setting.

\section{Materials and methods}

Computed tomographic scans were obtained from 152 orbits of 76 patients with Graves' ophthalmopathy. Some of these patients were included in prior studies. ${ }^{1-3}$ Their average age was $50 \cdot 1$ years (range, 12 to 82 ); there were 14 men and 62 women.

\footnotetext{
A PPARATUS

A GE 9800 or GE 8800 CT scanner was available for performing the orbital scans. In all but nine patients non-overlapping adjacent slices through the entire orbit (including roof and floor) were obtained in the axial plane at $1.5 \mathrm{~mm}$ intervals; in the nine patients

Correspondence to E Sverker Hallin, MD, Ogonkliniken, Sahlgrenska sjukhuset, S-413 45 Göteborg, Sweden.
}

slices were obtained at other intervals of $5 \mathrm{~mm}$ or less. The CT scans were imaged at window widths of 250 or 500 and at CT numbers of 0 to +20 Hounsfield units. These were quantitatively analysed with an image analyser.' An attached pen enabled contours to be traced and digitised for analysis. Packaged software provided with the image analyser was used to determine relative and absolute areas and volumes of structures by evaluating mid-orbital and serial twodimensional sections.

\section{PROCEDURE}

Axial CT scans were obtained at an angle of 0 to $-10^{\circ}$ relative to the canthal-meatal line. Gentle eye closure was used to limit volume averaging produced by eye movement during the scan and to prevent asymmetric extraocular muscle contraction that might increase apparent muscle thickness. The filmed image of each slice was traced precisely. The bony orbit, extraocular muscle, optic nerve, and globe were outlined. In addition the 'muscle area' and the 'preseptal area' of the mid-orbital axial CT section, defined as that section imaging the crystalline lens, the optic nerve head, and the optic canal, were calculated separately. The muscle area was defined as the sum of the medial and lateral areas. The preseptal area was defined as all tissue anterior to a line connecting the lateral orbital rims, limited by the soft tissue laterally and the orbital wall medially. A cross-section of this mid-orbital cut was defined as 


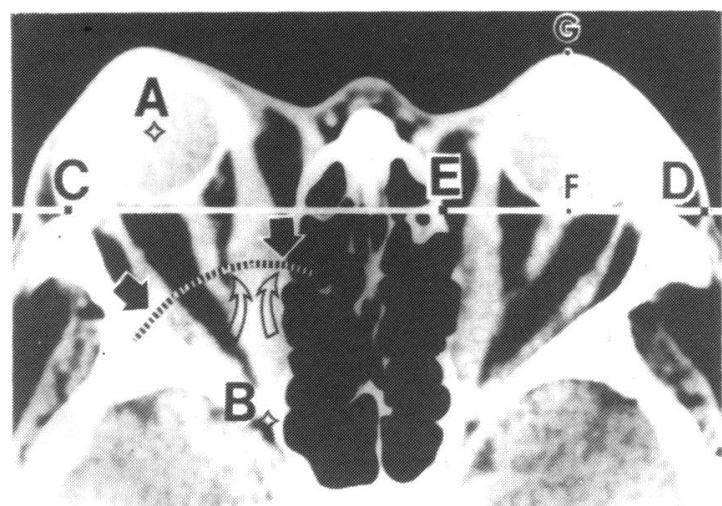

Fig. 1 Mid-orbital section of a CT scan in a patient with Graves'ophthalmopathy. Point A is the centre of the globe. Point $\mathrm{B}$ is the orbital apex. The dashed arcline is equidistant from point $\mathrm{B}$, including the midpoint of $\mathrm{AB}$. Along the arc, orbital width (black arrows), medial muscle width (open arrows), and intraconal fat width are measured. Line CD is defined by the anterior extent of the lateral bony orbital rim. Preseptal area is calculated to include all tissue anterior to line ED. Proptosis is defined as the distance FG.

an arc including the midpoint between the orbital apex and the geometric centre of the globe (Fig. 1). Along this cross-section, muscle width and the ratio of bony orbital width to intraconal fat width were calculated. Proptosis was defined as the distance between the apex of the cornea and a line connecting the lateral orbital rims as they appear on the midorbital axial section of the CT scan.

Orbital volumes were calculated by sequentially evaluating adjacent sections with the computed image analyser. Morphometric techniques were used

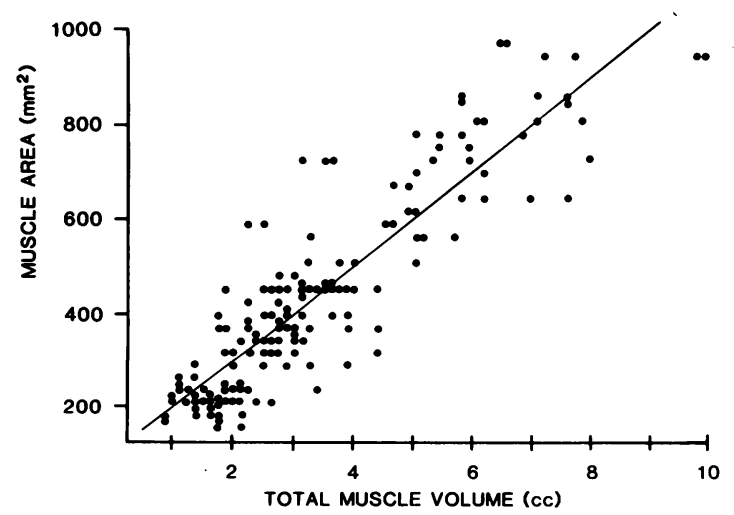

Fig. 2 Scatterplot comparing muscle area with total extraocular muscle volume. For this and all succeeding figures the solid line represents the least squares linear regression and the horizontal axis represents total extraocular muscle volume.
Table 1 Correlation of mid-orbital CT muscle areas with muscle volumes

\begin{tabular}{llll}
\hline Muscle area & $\begin{array}{l}\text { Total EOM } \\
\text { volume }\end{array}$ & $\begin{array}{l}\text { Horizontal EOM } \\
\text { volume }\end{array}$ & $\begin{array}{l}\text { Vertical EOM } \\
\text { volume }\end{array}$ \\
\hline Muscle area & $0 \cdot 89^{*}$ & 0.87 & 0.84 \\
Medial rectus & 0.88 & $0 \cdot 87$ & 0.81 \\
\hline
\end{tabular}

${ }^{*} \mathrm{p}<0.001$ for $\mathrm{r}>0.36$.

EOM=extraocular muscle.

that had previously been found to be accurate to within $7 \%$ to $10 \%$ for measurement of CT images. Errors due to volume averaging were minimised by using thin slices. In prior studies variation between individuals for singe muscles, estimated as the average ratio of single-muscle standard deviation to single-muscle mean volume, was $0 \cdot 35 .^{1-3}$

Because rectus muscles could not be reliably distinguished from adjacent extraocular muscles, the following pairs of muscles were analysed together: the levator and superior rectus, the superior oblique and medial rectus, and the inferior oblique and inferior rectus. The lateral rectus was analysed by itself. The volume of each muscle group was computed separately and compared with the overall volume of the orbit, after scaling the CT image so that the cross-sectional diameter of the globe on midorbital CT slice was made equal to $24 \mathrm{~mm}$. In this study each muscle is referred to by the included rectus muscle.

\section{Results}

COMPARISON OF MUSCLE AREA OF THE MIDORBITAL SECTION WITH EXTRAOCULAR MUSCLE VOLUME

A plot of total muscle area of the mid-orbital axial

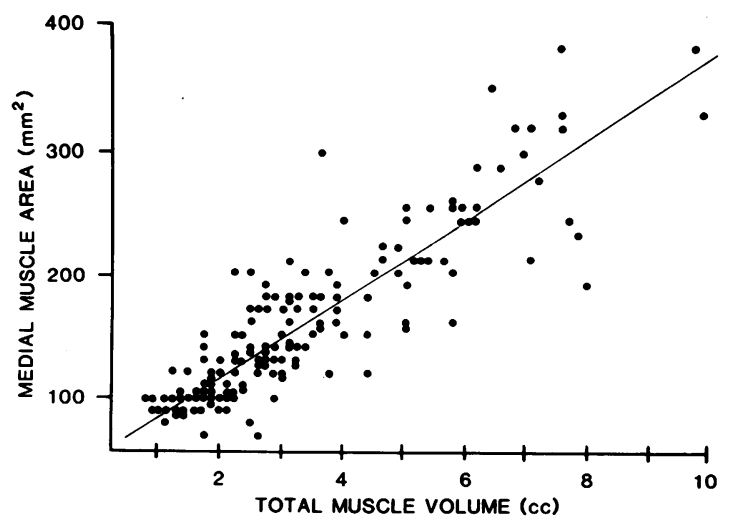

Fig. 3 Scatterplot comparing medial rectus muscle area with total extraocular muscle volume. 


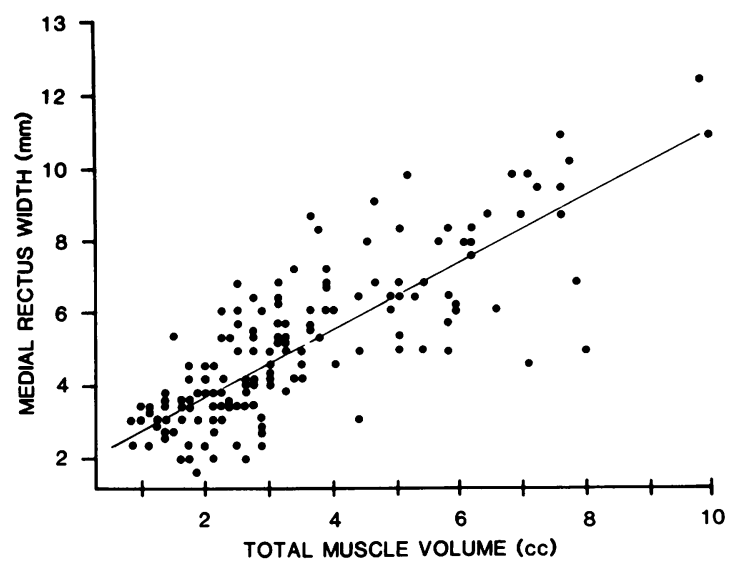

Fig. 4 Scatterplot comparing medial rectus muscle width with total extraocular muscle volume.

section ('muscle area') compared with total extraocular muscle volume is shown in Fig. 2. Least squares linear regression demonstrated a correlation between these two measures of $0 \cdot 89$. Correlations of muscle areas with total, horizontal, and vertical extraocular muscle volumes (all significant at $\mathrm{p}<0.001$ ) are summarised in Table 1 . A comparison of muscle area with horizontal muscle volume had a correlation of 0.87 , and that of muscle area with vertical muscle volume had a correlation of $0 \cdot 84$. In comparing the area of just the medial rectus to the total muscle volume, shown in Fig. 3, the correlation was 0.88 . Thus the area of the extraocular muscle, singly or together, imaged in the mid-orbital section appeared to reflect fairly accurately the total extraocular muscle volume.

\section{COMPARISON OF MID-ORBITAL MUSCLE AND BONY WIDTH WITH EXTRAOCULAR MUSCLE VOLUME}

Muscle width, a measure easier to obtain than orbital volume, was evaluated along an arc drawn through the mid-orbital point (Fig. 1). The plot of medial rectus muscle width compared with total extraocular

Table 2 Correlation of other measures obtained on midorbital CT slice to muscle volumes

\begin{tabular}{llll}
\hline Other measures & $\begin{array}{l}\text { Total EOM } \\
\text { volume }\end{array}$ & $\begin{array}{l}\text { Horizontal } \\
\text { EOM volume }\end{array}$ & $\begin{array}{l}\text { Vertical EOM } \\
\text { volume }\end{array}$ \\
\hline Medial rectus width & $0 \cdot 80^{*}$ & 0.79 & $0 \cdot 75$ \\
Preseptal area & 0.79 & 0.75 & 0.78 \\
Proptosis & 0.65 & 0.61 & $0 \cdot 63$ \\
Orbit/fat ratio & $0 \cdot 61$ & 0.65 & 0.52 \\
Intraconal width & $0 \cdot 16(\mathrm{NS})$ & - & - \\
\hline
\end{tabular}

${ }^{*} \mathrm{p}<0.001$ for $r>0.36$.

$\mathrm{EOM}=$ extraocular muscle.

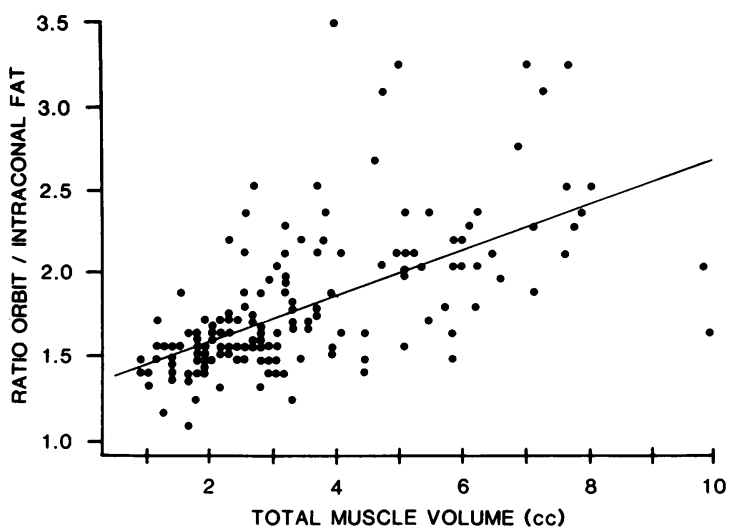

Fig. 5 Scatterplot comparing the orbit/fat ratio with total extraocular muscle volume.

muscle volume, shown in Fig. 4, gave a correlation of $0 \cdot 80$. Intraconal fat width was evaluated as a possible indirect measure of extraocular muscle encroachment on the optic nerve. However, the correlation with extraocular muscle volume was poor $(r=0 \cdot 16)$. The ratio of bony orbital width to intraconal orbital fat (orbit/fat ratio) along the arc subtended across the mid-orbital position was also calculated (Fig. 5), but had a better correlation $(0.61)$ with total extraocular muscle volume (Table 2 ).

\section{INDIRECT MEASURES OF EXTRAOCULAR MUSCLE} VOLUME

Periorbital swelling, known to correlate with extraocular muscle volume clinically, ${ }^{2}$ was identified on the CT scan by determining the area of the preseptal tissue on the mid-orbital section. Correlation between the preseptal area on a mid-orbital CT slice and total extraocular muscle volume, as plotted in Fig. 6, was $0 \cdot 79$.

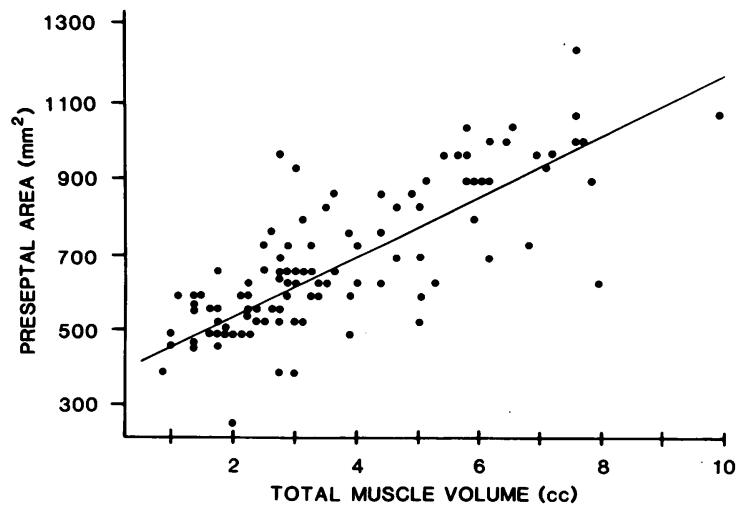

Fig. 6 Scatterplot comparing the preseptal area with total extraocular muscle volume. 
Proptosis, measured from the CT scans directly rather than obtained clinically, was evaluated as a possible measure of extraocular muscle volume; correlation was $0 \cdot 65$. Correlations of the indirect measures with horizontal and vertical extraocular muscle volume, as well as with total extraocular muscle volume (significant at $\mathrm{p}<0.001$ ), are summarised in Table 2.

\section{Discussion}

The principal alteration of the orbit in Graves' ophthalmopathy is enlargement of the extraocular muscles. This was first observed by Rundle and Pochin ${ }^{4}$ in cadavers and later confirmed both intraoperatively by Naffziger ${ }^{5}$ and radiologically by CT scan. ${ }^{1-36-9}$ The importance of extraocular muscle volume in causing optic neuropathy in Graves' disease has been emphasised ${ }^{6-8}$ and quantitatively assessed. ${ }^{1-39}$

Owing to the complexity and expense of obtaining and quantitatively analysing multiple thin axial CT sections, the development of alternative quantitative techniques for assessing the risk of optic neuropathy in Graves' ophthalmopathy is of clinical importance. ${ }^{23}$ In our radiographic study we looked at several measures of the orbital tissues that might correlate well with total extraocular muscle volume, but which would be easier to obtain either by computing tissue areas from a single axial section or by simply using a curvilinear measure at a fixed anatomical location within the orbit. Our results suggest that high correlations exist between a variety of mid-orbital tissue areas or linear measures and total extraocular muscle volume. Although some of these measures could be determined manually by the ophthalmologist, most CT scanners have software that can be used to map areas and calculate distances on single sections. Ideally radiologists could provide this clinically important, quantitative information to the ophthalmologist. Even proptosis and periorbital swelling may be more reliably estimated from the CT scan, because CT measures seem to improve the correlation of these measures with extraocular muscle volume substantially ( $r=0.65$ to 0.79 ) compared with their clinically obtained counterparts $(\mathrm{r}=0.29$ to 0.43$) .^{210}$

This study was supported in part by the Carmen and Bertil Regner's Fund, the Crown Princess Margareta's Fund, and by grant No. EY03908 awarded by the National Institutes of Health (USA).

ESH was a research fellow in the Department of Ophthalmology, University of Southern California School of Medicine.

\section{References}

1 Feldon SE, Weiner JM. Clinical significance of extraocular muscle volumes in Graves' ophthalmopathy: A quantitative computed tomography study. Arch Ophthalmol 1982; 100: 1266-9.

2 Feldon SE, Muramatsu S, Weiner JM. Clinical classification of Graves' ophthalmopathy: identiication of risk factors for optic neuropathy. Arch Ophthalmol 1984; 102: 1469-72.

3 Feldon SE, Lee CP, Muramatsu SK, Weiner JM. Quantitative computed tomography of Graves' ophthalmopathy: extraocular muscle and orbital fat in development of optic neuropathy. Arch Ophthalmol 1985; 103: 213-5.

4 Rundle FF, Pochin EE. The orbital tissues in thyrotoxicosis: a quantitative analysis relating to exophthalmos. Clin Sci 1944; 5: 51-74.

5 Naffziger HC. Pathologic changes in the orbit in progressive exophthalmos, with special reference to alterations in the extraocular muscles and the optic disks. Arch Ophthalmol 1933; 9: $1-12$.

6 Trokel SL, Hilal SK. Submillimeter resolution CT scanning of orbital diseases. Ophthalmology 1980; 87: 412-7.

7 Kennerdell JS, Rosenbaum AE, El-Hoshy MH. Apical optic nerve compression of dysthyroid optic neuropathy on computed tomography. Arch Ophthalmol 1981; 99: 807-9.

8 Trokel SL, Jakobiec FA. Correlation of CT scanning and pathologic features of ophthalmic Graves' disease. Ophthalmology 1981; 88: 553-64.

9 Hallin S. Distribution of extraocular muscle swelling in endocrine exophthalmos. Proc VII Congr Eur Ophthalmol (Helsinki) 1985: 528-9.

10 Hallin ES, Feldon SE. Graves' ophthalmopathy: II. Correlation of clinical signs with measures derived from computed tomography. Br J Ophthalmol 1988; 72: 678-82.

Accepted for publication 2 July 1987. 